\title{
AN ANTERIOR APPROACH FOR RESECTION AND ADVANCEMENT OF THE LEVATOR*
}

\author{
BY \\ R. DALGLEISH
}

Department of Ophthalmology, University of Manchester

RESECTION and advancement of the levator palpebrae superioris gives the best functional and cosmetic result of any ptosis operation. As a rule, operation on the levator is indicated if there is a degree of function in this muscle. Myasthenia gravis, the myopathies, jaw-winking, and related anomalies of innervation are the exceptions to this rule.

Bowman was perhaps the first to attempt correction of ptosis by resection of the levator, either by the conjunctival or by the skin approach (Bader, 1857). Nothing can be achieved by the posterior approach that cannot be achieved by the anterior approach. The latter is particularly indicated when the levator action is poor. It is the purpose of this paper to describe a simple technique for resection and advancement of the levator by way of an anterior approach.

Surgical Anatomy (Fig. 1, opposite).-The septum orbitale is loosely attached to the posterior surface of the orbicularis, from the superior orbital margin down to the lashes; anterior to the tarsus it is very thin, but above it is thicker and more easily identified (Berke, 1957). At the level of the palpebral sulcus, the orbital septum is largely reflected back along the upper surface of the levator aponeurosis, to become continuous with the sheath of the levator. This reflection of the orbital septum forms a cul-de-sac which is filled with orbital fat.

The main attachment of the levator is to the skin and subcutaneous tissues at and below the level of the palpebral sulcus. This attachment is achieved by successive layers of fibres of the levator aponeurosis, which pass through the arcades of the palpebral portion of the orbicularis oculi. There is a weak attachment of the levator to the anterior surface of the tarsal plate. Müller's muscle is inserted into the upper edge of the tarsus. The conjunctiva is closely applied to the posterior surface of the tarsal plate and Müller's muscle, with very little intervening connective tissue.

The standard anterior approach is via an incision $6 \mathrm{~mm}$. above and parallel to the lid margin, for nearly the entire length of the tarsal plate (Stallard, 1958). The anterior surface of the tarsal plate is then exposed along the length of this incision. In order to expose the anterior surface of the levator, the dissection must now proceed upwards. This presents some difficulty: (1) If the dissection proceeds on the same plane as the anterior surface of the

* Received for publication June 28, 1962. 
Fig. 1.-Vertical section through upper lid.
A. Orbital septum
B. Fat pad
C. Levator aponeurosis
D. Müller's muscle
E. Tarsal plate

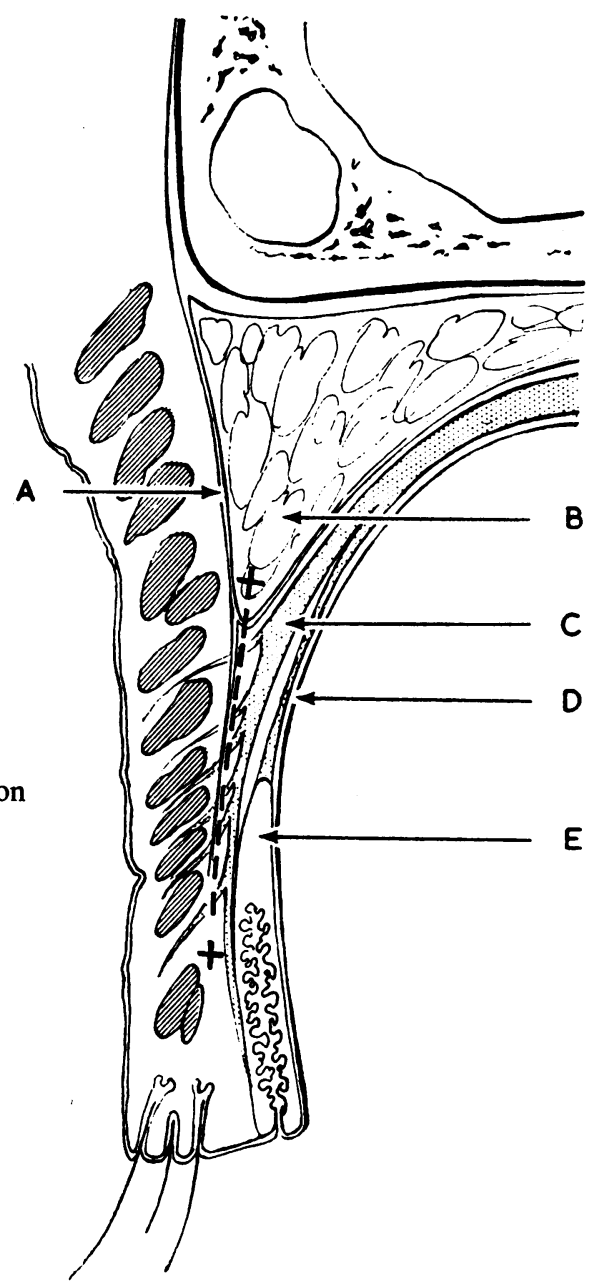

tarsal plate, the space between the levator and Müller's muscle will be entered, and the posterior surface of the levator will be exposed.

(2) If the dissection upwards is too anterior, the palpebral arcades of the orbicularis will be damaged, and some will be resected with the levator; this may lead to defective closure post-operatively.

(3) The correct line of the dissection is indicated in Fig. $1(X----X)$. Inferiorly, the anterior surface of the tarsal plate is the essential landmark and starting point. Superiorly, the orbital fat-pad is the landmark. It is essential to expose this, because the fat is visible only when the orbital septum has been incised, and failure to do so may lead to resection of the septum instead of the levator. Fig. 1 indicates that there is no tissue plane connecting the two landmarks; active dissection across tissue planes is necessary. The fibres of the levator aponeurosis tend to direct one into the substance of 
this muscle when proceeding from below upwards. The dissection is more easily achieved proceeding from above downwards with the anterior surface of the levator as the starting point. A technique will be described, whereby the dissection is carried out in this direction.

As a final application of the surgical anatomy, it will be appreciated that it is difficult to enter the plane between the conjunctiva and Müller's muscle via an anterior approach. A technique will be described which facilitates this manoeuvre.

\section{The Operation}

General anaesthesia is preferred. The junctions of the middle third with the medial and lateral thirds of the lid margin are marked by vertical strokes of gentian violet on the lash line. Two lines are now drawn parallel with the lid margin. The first is $6 \mathrm{~mm}$. above the margin, extending for nearly the entire length of the tarsal plate. The second is $16 \mathrm{~mm}$. above the margin; this line is about $6 \mathrm{~mm}$. long and is situated centrally on the lid (Fig. 2).

Two traction sutures are placed through the lid margin at the marked junctions of the central with the medial and lateral thirds. A lid guard is inserted. An incision to the depth of the subcutaneous tissue is made along the entire length of the lower horizontal line. A short incision is made along the length of the upper horizontal line. Using pointed straight scissors, with the blades held parallel to the roof of the orbit, this short incision is deepened through the fibres of the orbicularis, through the orbital septum, and into the fat-pad above the levator (Fig. 3). The yellow fat is easy to recognize, and it will tend to herniate through the incision if backward pressure is applied. The slightly curved blade of a flat dissector (Inset Fig. 4) is now inserted backwards and downwards into the fat-pad via this incision. It is important to keep the flat surface of the dissector firmly in contact with the anterior surface of the levator. In order to achieve this, the lid guard is held in one hand, and the dissector in the other. While exerting firm backward pressure, short horizontal movements are executed with the dissector proceeding in a downward direction. In this way, the desired plane between the orbicularis anteriorly and the levator posteriorly will be opened (Fig. $1, X---X$ ). It must be emphasised that firm active dissection is required, as the "orbital septum cul-de-sac" must be opened, and the successive layers of fibres of the levator aponeurosis passing forwards through the orbicularis must be cut across. Having executed a few strokes with the dissector as described, it will be seen that the blade of the instrument has passed below the line of the horizontal incision $6 \mathrm{~mm}$. above the lid margin. This incision is now deepened onto the blade of the dissector along its entire length (Fig. 4). The upper skin fold is retracted, and the complete anterior surface of the levator is exposed to view. On average, it takes 3 to 4 minutes from the commencement of the skin marking to the completion of this stage of the operation. The 
FIG. 2
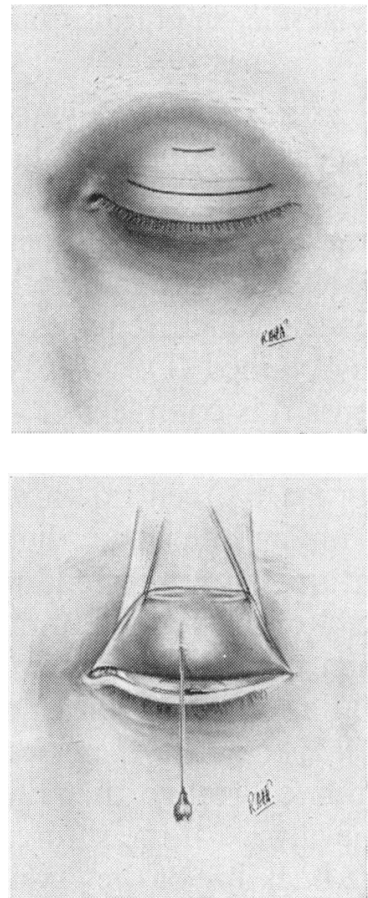

FIG. 5
Frg. 3
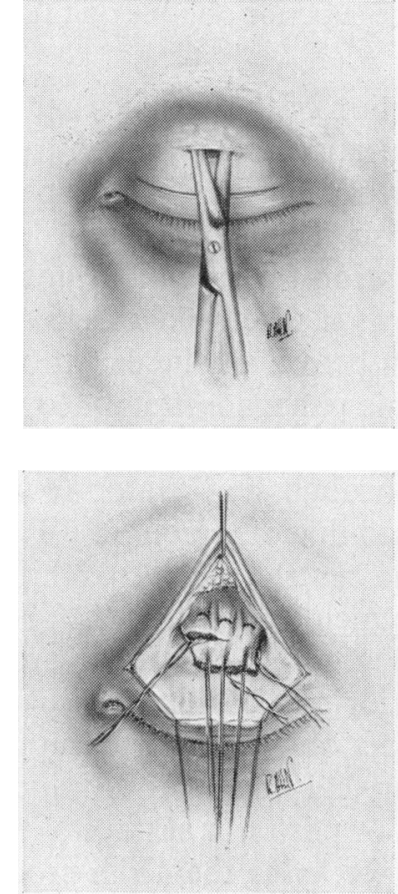

FIG. 6
FIG. 4
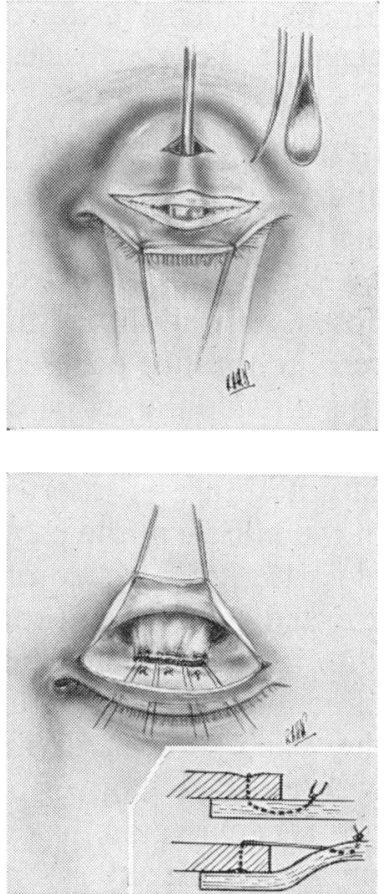

Fig. 7

anterior surface of the tarsal plate is now exposed, the lower skin fold is retracted, and haemostasis is obtained.

The next step is to open up the plane between the conjunctiva and Müller's muscle, thereby separating these two structures. Various techniques have been employed by different surgeons in order to achieve this. The following technique is quick and effective.

The lid guard is withdrawn from under the upper lid, and a clean guard is inserted from above into the wound. Using the traction sutures through the lid margin, the lid is everted over the guard. Saline is then injected subconjunctivally at the upper edge of the tarsal plate (Fig. 5). This injection may be repeated at more than one point until the conjunctiva is well raised from the underlying muscle. The guard is then removed from the wound and re-inserted into the conjunctival sac, thereby causing the lid to revert to the normal position. A short vertical incision is made centrally through the sheath of the levator, slightly above the upper edge of the tarsal plate. This is deepened with pointed scissors, through the substance of the levator and Müller's muscle, into the saline-filled subconjunctival space. Using the narrow curved blade of a flat dissector, the separation of the conjunctiva is carried medially, laterally, and upwards. Three traction sutures are inserted through the levator and Müller's muscle; these muscles are then cut free above the upper edge of the tarsal plate. The muscles are hinged forward, and the separation of the conjunctiva is carried upwards to the superior limit of the 
fornix. The medial and lateral check ligaments of the levator are severed, taking care to avoid the tendon of the superior oblique on the medial side, and the lacrimal gland on the lateral side.

The next step is the insertion of the sutures that will fix the resected levator to the tarsal plate. The question of how much to resect is the most variable and difficult problem in ptosis surgery. Pre-operatively, an assessment is made of the amount of ptosis and the degree of levator function as outlined by Berke (1957). At operation the "quality" and mobility of the muscle is noted. In addition, there is a threshold of effectiveness, which requires a certain amount of resection before any lid elevation is obtained (Fox, 1961). It is outside the scope of this paper to attempt to discuss this complexity.

Having decided on the amount to be resected, a double-armed 4.0 chromic catgut suture is inserted through the levator to embrace the central third of the muscle at the proposed line of resection. Two similar sutures are then placed on either side of the central suture. Whip sutures are preferred, as knotted sutures tend to "bunch" the muscle. The levator is excised $3 \mathrm{~mm}$. anterior to these sutures (Fig. 6). The sutures are then placed in a vertical direction through the anterior half of the tarsal plate at appropriate sites, so that each suture will support one-third of the upper lid: they are then tied with the first loop of a surgical knot. It is important to arrange that the sutures emerge on the deep or posterior surface of the levator. If the sutures emerge on the anterior surface, tying them will produce buckling of the tarsal plate, with associated post-operative ectropion (Inset Fig. 7). At this stage the lid guard is withdrawn, and careful attention is given to the elevation and curve of the upper lid margin. Undercorrection is remedied by arranging another line of fixation sutures to replace the first, so that more muscle can be resected where required. Overcorrection is remedied by loosening one or more of the fixation sutures, and allowing the levator to retract at those points. It is well worthwhile spending extra time at this stage of the operation, and the second loops of the surgical knots should not be tied until one is completely satisfied with the result. This facility for adjustment is one of the great advantages of the anterior approach.

Having fastened the levator to the tarsal plate, four or five interrupted 000 silk sutures are placed through the muscle just above the line of resection. These sutures emerge through the skin at intervals adjacent to the lash line (Fig. 7). They are tied with sufficient tension to produce a minimal degree of detraction of the lid margin from the underlying globe. Their purpose is to prevent post-operative downward tilting of the lashes when the lid is elevated. This complication is quite common, and may amount to a frank trichiasis if these sutures are omitted.

The long horizontal skin incision is now closed with interrupted vertical mattress sutures of 000 black silk. The deep loop of these sutures passes through the underlying levator, in order to create an upper lid fold. A single suture closes the short horizontal skin incision. 
Antibiotic ointment is instilled into the conjunctival sac. 'Tulle gras, followed by a dental roll of compressed wool, is placed over the skin incision. Two traction sutures through the lower lid are carried up over this roll to -be secured to the forehead with adhesive tape. A compression dressing is applied.

\section{Summary and Conclusions}

The anatomy of the upper lid relating to surgery to the levator palpebrae superioris is discussed.

An anterior approach for resection and advancement of this muscle is described.

It is claimed that this approach overcomes some of the technical difficulties encountered with the standard anterior approach. To date, it has been used in fifteen cases, and has resulted in reduced operating time and greater clarity of dissection. By virtue of its size and position, the additional skin incision required for this approach does not produce any cosmetic blemish.

The illustrations were drawn by Mr. R. L. Neave. Medical Artist, Manchester University, whose skill and co-operation I gratefully acknowledge. The soft-tissue dissector used in this operation was made to the author's specification by C. W. Dixey and Son Ltd., London, W.1.

\section{REFERENCES}

Bowman, W. P. (1857). In Bader's Report in Ophthal. Hosp. Rep., 1, 33.

BERKE, R. N. (1957). In "Management of Complications in Eye Surgery", ed. R. M. Fasanella, chap. 5, p. 71. Saunders, Philadelphia.

Fox, S. A. (1961). Arch. Ophthal. (Chicago), 65, 345.

Stallard, H. B. (1958). "Eye Surgery", 3rd ed., p. 158. Wright, Bristol. 